\title{
Computational Modeling of Pool Games: Sensitivity of Outcomes to Initial Conditions
}

\author{
Christian Leerberg and M.W. Roth \\ Department of Physics \\ University of Northern lowa \\ Cedar Falls, lowa 50614-0150 USA
}

Received:April 4, $2002 \quad$ Accepted: September 19, 2002

\begin{abstract}
We present a study of the sensitivity of trajectories of pool balls to initial conditions. In the first component of the study our simulations include all sixteen balls. Variables include cue ball initial velocity and position on the "table". We find that in a certain regime of initial conditions the system seems to show self-similarity, but as the range of initial cue ball angle and initial velocity is restricted, the system exhibits an interesting evolution towards a single point in parameter space, with the ball landing in only one pocket. We also examine the effects of varying the number of balls on the table, and how their dynamics may be interpreted using various plots and maps. Finally, the trajectory of a single cue ball is examined while it moves through the table space. Starting with the cue ball placed in the middle of the right wall of the table (traditional and rectangular in shape) and fired directly downward the system exhibits a two-cycle pattern. Then as the angle of fire is increased the system exhibits a four cycle, a three cycle and finally a two cycle all separated by noisy patterns. Effects of numerical artificialities are briefly discussed.
\end{abstract}

\section{INTRODUCTION}

Chaos is derived from the Greek word for "abyss" and may be translated to mean "where chance is supreme". In fact, popular notions equate chaos with randomness, which is not at all the true physical and mathematical definition. Chaos (often "deterministic chaos") is the physical manifestation of sensitivity of a particular set of system outcomes to another set of its initial conditions ${ }^{1}$. Since the introduction of chaos into the field of physics many systems have been studied in order to understand disorder: e.g., cloud formation, weather patterns, and the stock market. The game of pool has also been studied on a number of levels for geometrical, physical, and chaos-related reasons. There are a wide variety of pool simulators ${ }^{2-5}$ whose chief purpose is to entertain. Such simulators strive, with varying degrees of accuracy, to be as realistic as possible, including the effects of friction, spin, and non-ideal ball and bumper collisions. Although intricately programmed, enjoyable and in many cases customizable, such simulators lend themselves quite poorly to innovative scientific study due to the inability of a user to vary the model parameters and adjust the numerical output in novel ways. There are algorithms ${ }^{6,7}$ which model the behavior of one ball on tables whose boundaries have varying geometries and, in both visual and numerical settings, follow the balls through phase space and track the divergence of paths for initial conditions that could, in principle, start out arbitrarily close.

The purpose of this study is to formulate a reasonably realistic model of pool balls on a typical table and to have the ability to study the sensitivity of the system to initial conditions over a wide range of system parameters. By utilizing different output representations we may easily vary the algorithm to incorporate many different scenarios. The task at hand is largely theoretical, while relying on experimental results for correspondence with actual data. Since much of the results are found by computation, we are also concerned with how the results depend on numerical 
artificialities and how much are real physics. Such considerations are paramount in interpreting our results.

\section{THEORY AND COMPUTATIONAL METHOD}

Our model is classical in that Newton's laws of motion govern its time evolution. As such, our double-precision FORTRAN algorithm steps the system through time, and therefore determination of the forces acting on the balls is central to the success of our endeavor. We can easily ignore gravitational interactions between the balls but three categories of interactions are identified as playing key roles: ball-table, ball-air and ballball.

Ball-table interactions include friction, spin dampening and reflection at boundaries. The type of friction considered is not the static friction that results in rotation but an effective kinetic friction that results in damping of rectilinear center-of-mass motion: $\vec{f}_{i}=-\mu_{k} m_{i} g \hat{v}_{i}$. Here $\mathrm{m}_{\mathrm{i}}$ is the mass of the ball, $g$ is the acceleration of gravity and $\hat{v}_{i}$ is a unit vector in the direction of the velocity of ball (i). The coefficient of friction is measured experimentally and taken to be the same for all balls; important parameters used in this study are shown in Table 1. Balls may also be assigned spin angular momentum directed perpendicular to the table:

$$
\vec{S}_{i}=S_{i 0} e^{-\alpha t} \hat{z} .
$$

Equation 1 reflects the fact that the spin of a particular ball damps with time due to dissipative forces. The constant $\alpha$ may be measured experimentally when it is to be included in a simulation by fitting a curve of the form in eqn. (1) to the rotational motion of real pool balls. No spin is used for the simulations presented in this paper. This spin does not cause changes in the centerof-mass motion of a particular ball unless it either reflects off a boundary or collides with another ball. For reflection of ball (i) we have

$$
\begin{gathered}
v_{i x}^{\prime}=v_{i x}+\beta \hat{v}_{i} \times \hat{S}_{i} \\
v_{y}^{\prime}=-v_{y}
\end{gathered}
$$

for a horizontal wall (top or bottom of the table) and

$$
\begin{gathered}
v_{y}^{\prime}=v_{y}+\beta \hat{v}_{i} \times \hat{S}_{i} \\
v_{x}^{\prime}=-v_{x}
\end{gathered}
$$

for a vertical wall (left or right boundaries) and both conditions simultaneously if the ball hits a corner. Here the constant $\beta$ reflects to what degree the ball's spin couples with the edges of the table and, like $\alpha$, can be measured experimentally by fitting equations (2a) and (2c) to the trajectories of real pool balls impacting the table walls with known spins. Using arguments of ball and table uniformity $\beta$ (when included) may be taken to be the same for all balls. The program was tested with a value of 0.1 but this parameter was not used in the simulations presented here. The primed velocities represent those after the encounter and the unprimed ones are preencounter velocities.

The only ball-air interaction is taken to be drag resistance,

$$
\vec{F}_{i D}=-\frac{1}{2} C_{D} \rho \pi r_{i}^{2} v_{i}^{2} \hat{v}_{i} .
$$

Here $\rho$ is the density of the air, $r_{i}$ is the radius of the ball, $C_{D}$ is the drag coefficient, $v_{i}$ is the speed of the ball and $\hat{v}_{i}$ is a unit vector if the direction of the ball's velocity which, when coupled with the negative sign in the equation, indicates that this force always acts in opposition to the ball's motion.

After the forces on all $\mathrm{N}$ particles are calculated, the system is advanced in time utilizing a simple forward-difference scheme:

$$
\begin{gathered}
\vec{r}_{i}(t+\Delta t)=\vec{r}_{i}(t)+\vec{v}_{i}(t) \Delta t+\frac{1}{2} \frac{\vec{F}_{i}}{m_{i}}(\Delta t)^{2} \\
\vec{v}_{i}(t+\Delta t)=\vec{v}_{i}(t)+\frac{\vec{F}_{i}}{m_{i}} \Delta t .
\end{gathered}
$$

The time step $\Delta t$ in the simulation is chosen such that any physical overlap of two pool balls is small compared to either of their dimensions. After the system has advanced, the algorithm considers ball-ball interactions. 


\begin{tabular}{|c|c|c|}
\hline Parameter & Symbol & Value \\
\hline Cue ball mass & $\mathrm{m}_{1}$ & $1.6 \times 10^{-1} \mathrm{~kg}$ \\
\hline Cue ball radius & $\mathrm{r}_{1}$ & $2.79 \times 10^{-2} \mathrm{~m}$ \\
\hline Ball mass & $\mathrm{m}_{2}-\mathrm{m}_{16}$ & $1.7 \times 10^{-1} \mathrm{~kg}$ \\
\hline Ball radii & $\mathrm{r}_{2}-\mathrm{r}_{16}$ & $2.86 \times 10^{-2} \mathrm{~m}$ \\
\hline Coefficient of friction & $\mu_{\mathrm{k}}$ & 0.027 \\
\hline Horizontal table dimension & $\mathrm{A}$ & $2.6 \mathrm{~m}$ \\
\hline Vertical table dimension & $\mathrm{B}$ & $1.3 \mathrm{~m}$ \\
\hline Drag coefficient & $\mathrm{C}_{\mathrm{D}}$ & 0.5 \\
\hline Acceleration of gravity & $\mathrm{g}$ & $9.8 \mathrm{~m} / \mathrm{sec}^{2}$ \\
\hline Time step & $\Delta \mathrm{t}$ & $10^{-3} \mathrm{sec}$ \\
\hline Density of air & $\rho$ & $1.29 \mathrm{~kg} / \mathrm{m}^{3}$ \\
\hline
\end{tabular}

Table 1. Important parameters used in the simulation. All measurements were made by the authors on a pool table in the student union at the University of Northern lowa, and the dimensions of the table are not referenced explicitly in any of the equations.

It sorts through all balls and determines which groups of balls were not initially touching and are now overlapping because of true collisions. A ball may collide with a group of balls already touching. In that case, the algorithm expands all the ball pairs $(i, j)$ that are touching along $\vec{r}_{i j}$ (to eliminate overlap) and then treats the collision as a series of virtual collisions which propagates by involving consecutive pairs of masses $(i, j)$ overlapping in that group in a sequence:

$$
\begin{gathered}
\vec{v}_{i}^{\prime}=\vec{v}_{i}+\left(\frac{(1+e) m_{j}}{m_{i}+m_{j}}\right) \frac{\left(\vec{v}_{i j} \bullet \vec{r}_{i j}\right)\left(\vec{r}_{j}-\vec{r}_{i}\right)}{r_{i j}^{2}}+\gamma\left(\hat{S}_{i}+\hat{S}_{j}\right) \times \hat{r}_{i j} \\
\vec{v}_{j}^{\prime}=\vec{v}_{j}-\left(\frac{(1+e) m_{i}}{m_{i}+m_{j}}\right) \frac{\left(\vec{v}_{i j} \bullet \vec{r}_{i j}\right)\left(\vec{r}_{j}-\vec{r}_{i}\right)}{r_{i j}^{2}}-\gamma\left(S_{i}+S_{j}\right) \times \hat{r}_{i j} .
\end{gathered}
$$

Here $\vec{v}_{i j}$ is the velocity of ball (j) relative to that of (i) and $e$ is the elasticity of the collision, which can be taken to range anywhere from $e=1$ to $e=0.1 ; e=1$ is used in the work presented here (enabling us conduct runs with rather long simulated times) but $e<1$ was used to test the program. The parameter $\gamma$ is an experimentally measured constant that describes the strength of the spin-spin coupling. The system is not allowed to advance in time unless a configuration is reached where a virtual collision does not result in particle overlap; this configuration is taken to be the real outcome of the collision. It is well known that uncertainty in the 
outcome of the simulation may arise when more than two balls collide and touch at once $^{8}$, which could also be the case. Since the algorithm searches for touching pairs, virtual collisions still proceed at different times when three or more balls collide at once. Such artificial delay introduces uncertainty in the outcome of the simulation and this effect must be alleviated. The postcollision velocities are therefore calculated as averages of the velocities obtained when the algorithm searches once in forward order and once in reverse order. The method used was validated by testing it in situations involving simple geometries where the results are known: one- and twodimensional arrays of interacting and noninteracting masses undergoing collisions of varying elasticity - a mass colliding with two masses already touching for example.

Various methods are undertaken to rule out unimportant variables but still have the theory reflect what is encountered in the real world. For example, the simulation is run including all the types of interactions described earlier. However, for much of the computation even a very small friction coefficient or air resistance reduces the velocity of the pool balls enough to stop motion before conclusive results can be reached. Due to this, air resistance and friction are eliminated for much of the study. In addition, our efforts to attempt an understanding of the system's long-time behavior preclude the use of any spin for the time being. It is important at this point to mention that we typically would run the program for hundreds of thousands to tens of millions of time steps, corresponding to a simulated time ranging between about 100 seconds and 28 hours which take anywhere from 5 minutes to 16 hours using an 866 $\mathrm{MHz}$ Pentium III processor. Thus, even a minute friction coefficient $(\mu=.05$, for example, similar to a smooth ski on ice) easily brings the system to a halt by depleting its kinetic energy.

We conducted simulations with varying numbers of balls $(\mathrm{N}=16$ and $\mathrm{N}=3)$, as well as with only the cue ball. In the former type of simulations there are six pockets placed at the positions of real pockets on traditional pool tables. The simulated pockets are semicircles of radius $5 \mathrm{~cm}$ and are numbered from 1 to 6 . A ball "falls into a pocket" when reflecting from the wall such that its geometrical center lies within the pocket semicircle. Initial conditions include cue ball speeds in a range centered about $3 \mathrm{~m} / \mathrm{sec}$ and velocity angles centered about $\vartheta_{0}=0^{\circ}$. The simulations with multiple balls are run out to $10^{8}$ time steps to ensure that most of the balls ended up in pockets. For simulations involving only the cue ball, however, the pool table perimeter is divided up into 300 "pockets" of equal size, beginning with 1 at the upper left-hand corner and running clockwise to 300 . In the simulations involving only the cue ball, the ball was not allowed to fall in any of the pockets. Our simulations involving only the cue ball were run out to $3 \times 10^{7}$ time steps. The ball is placed in the middle of the right side of the table with an initial speed of $3 \mathrm{~m} / \mathrm{sec}$ and a velocity angle that varied from the ball being hit straight down to it being hit directly to the left.

\section{RESULTS}

Figures 1 and 2 show Kelly plots for ball 13 with various initial cue ball speeds and angles. Inspired by the artwork of Ellsworh Kelly, they can serve as a reasonable visual test for such physical processes as intermittency, Gaussian/uniform randomness, and Brownian motion to name a few. Kelly plots show a sequence of parameters that are binned and subsequently assigned numbers, which are then plotted as different colors. For our work we assigned a shade of gray to the number of the pocket a particular ball lands in (ball 13 in this case) after $3 \times 10^{6}$ time steps versus the angle at which the cue ball approaches the rack (x-axis) and the velocity at which it initially travels (y-axis).

Figure 3 shows return maps as well as histograms for the cue ball in simulations involving only three balls (top two plots) and all 16 balls (bottom two plots) for a simulation ran for $10^{7}$ steps. For a certain parameter of the system taking on values $\left\{x_{1}, x_{2}, x_{3}, \ldots\right\}$ the return map has points $\left(x_{1}, x_{2}\right),\left(x_{2}, x_{3}\right)$, etc. In our case, the variable $x$ is the point along the perimeter of the table where the ball reflects. Since the return map involves one position and the one immediately subsequent, it gives an indication as to the memory of the system. If the return map lies along the graph of a 

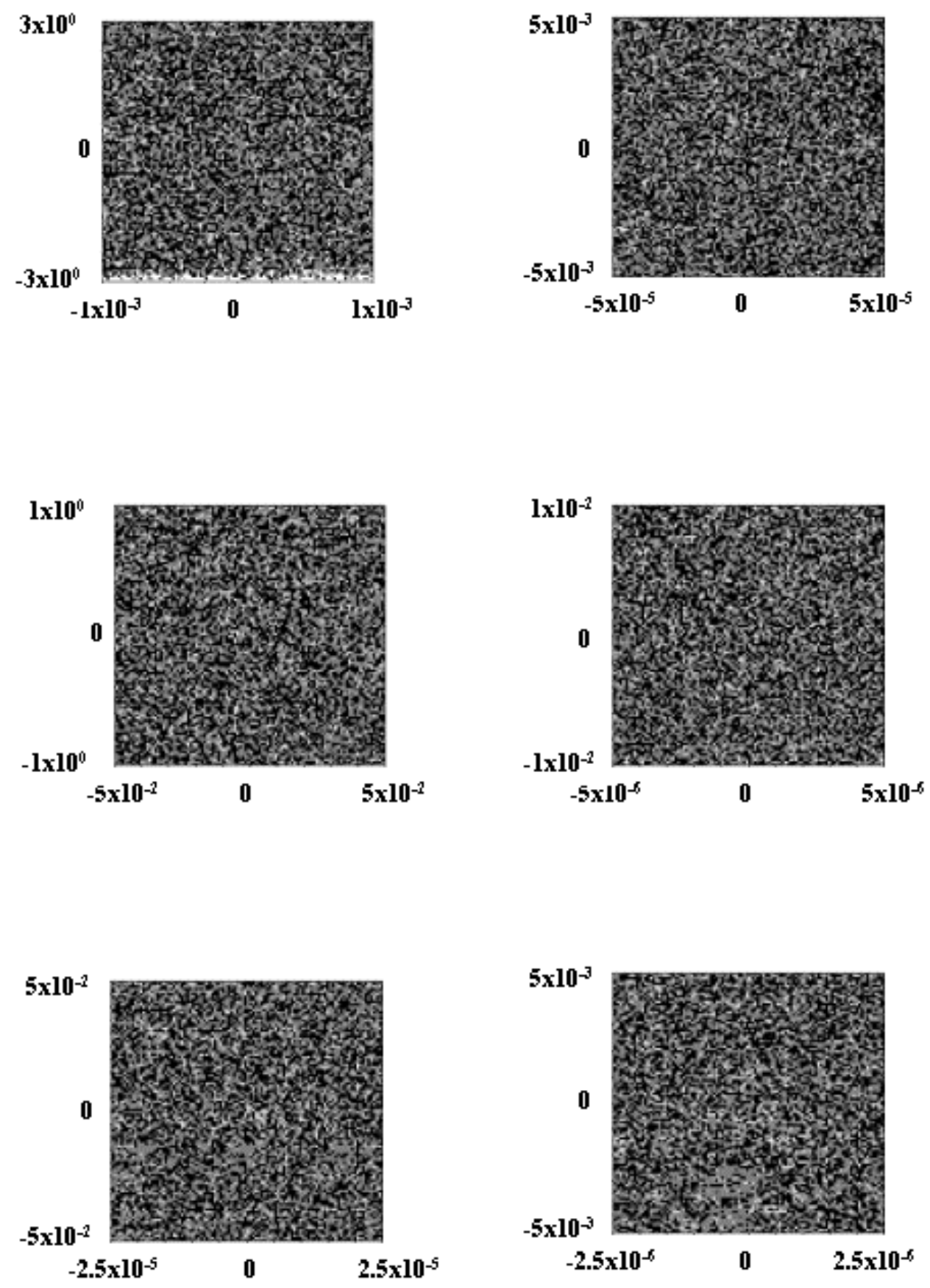

Figure 1. Kelly plots for ball 13 with various initial cue ball velocities and angles. The vertical axes are $\left(\mathrm{v}_{0}-3 \mathrm{~m} / \mathrm{sec}\right)$ and the horizontal axes are initial cue ball velocity angle in degrees. All six pockets have different numbers, and the grayscale varies according to the pocket that the ball ultimately lands in. Note that the range of initial conditions tend to be restricted from upper left to lower right, over a wide range of initial conditions. 
function then the parameter at each point depends only on the one before it and only one step is involved in determining the future of the system; more elaborate return maps suggest longer system memories. For histograms, a certain parameter is divided into bins and every time that parameter takes on a certain bin value, the counter for that bin is increased by one. Our histograms record the frequency of occurrence of reflection of the cue ball from a given point along the perimeter of the table. Histograms are designed to give an idea of the amount of time the system spends in certain regions of phase space - points along the table perimeter in our case. Histograms are also useful in determining if the system exhibits any $n$-fold periodic motion in phase space: a perfect $n$-cycle ( $n$ being an integer greater than zero) or possibly in a noisy n-cycle. Figures 4-6 show return maps (left) and histograms (right) for the cue ball in simulations involving only the cue ball for various initial conditions.

Bifurcation occurs when the behavior of a system changes suddenly for a small change in some parameter, and is a hallmark of chaotic behavior. Figure 7 is a bifurcation diagram for simulations involving only the cue ball. Bifurcation diagrams plot the certain position values (on the $y$-axis) versus a chosen parameter (on the $\mathrm{x}$-axis). Our bifurcation diagrams plot the position along the perimeter of the table the cue ball reflects from along the $y$-axis vs. its initial velocity angle on the $x$-axis. So if a bifurcation diagram is scanned vertically, a histogram is recovered, only lacking information about relative peak intensity. Bifurcation diagrams are useful in understanding how the system dynamics changes with $\mathrm{x}$-axis parameter value.

\section{DISCUSSION}

\section{a. Simulations with 16 balls}

After running the program numerous times and examining the results of each of the runs we conclude that there is indeed a profound effect of initial conditions on the final outcome of the system. Of all our results, the most basic and information-rich analysis tools are Kelly plots. In Figure 1 it appears that they show signatures of some type of randomness. In addition, they appear to exhibit self-similarity, a hallmark of chaotic systems-appearing the same over a wide range of parameter space. Figure 2 shows that as the phase space range of initial conditions constricts, the system shows a complicated evolution from an apparently random, noisy pattern to a single color (single pocket) plot.

An anomaly occurred during the computational process that warrants some attention. After studying the Kelly plots it became clear that a pattern was present in the form of vertical demarcations (seen in Figure 2). Much debate went into the question of whether this vertical structure is due to artificialities of computation or due to real physics. Eventually, the answer was found, and the results worth mentioning here. For small angles $\vartheta$ in radians the sine function can be approximated by $\sin \theta \approx \theta$ and the cosine function by $\cos \theta \approx 1-\frac{\theta^{2}}{2}$. So, even in double precision, a point is reached at small enough initial cue ball velocity angles where the sine function is correctly computed but the cosine function is not because the actual angular dependence on the angle is so small compared to unity that round-off error occurs. Hence, the cosine function does not continuously vary with theta but behaves rather like a step function in regions of very small angle. At any rate the system seems to exhibit strong sensitivity to initial conditions up until the point at which the ball lands in only one pocket (when the range of initial conditions is very restricted).

\section{b. Simulations with varying numbers of balls}

The histograms in Figure 3 do not seem to be very useful, with the exception that they confirm that it is highly unlikely that any balls will strike the corners of the table. The return maps, on the other hand, tell much about the dynamics of the system. Note, for example, that the plot for $\mathrm{N}=3$ is much denser than that for $\mathrm{N}=16$. This is a direct result of the fact that there are more ball-ball collisions for $N=16$ and hence less chance for the ball to strike a "pocket" directly after having hit another pocket. Note also that there are vertical and horizontal lines that are un-populated; such lines correspond to 

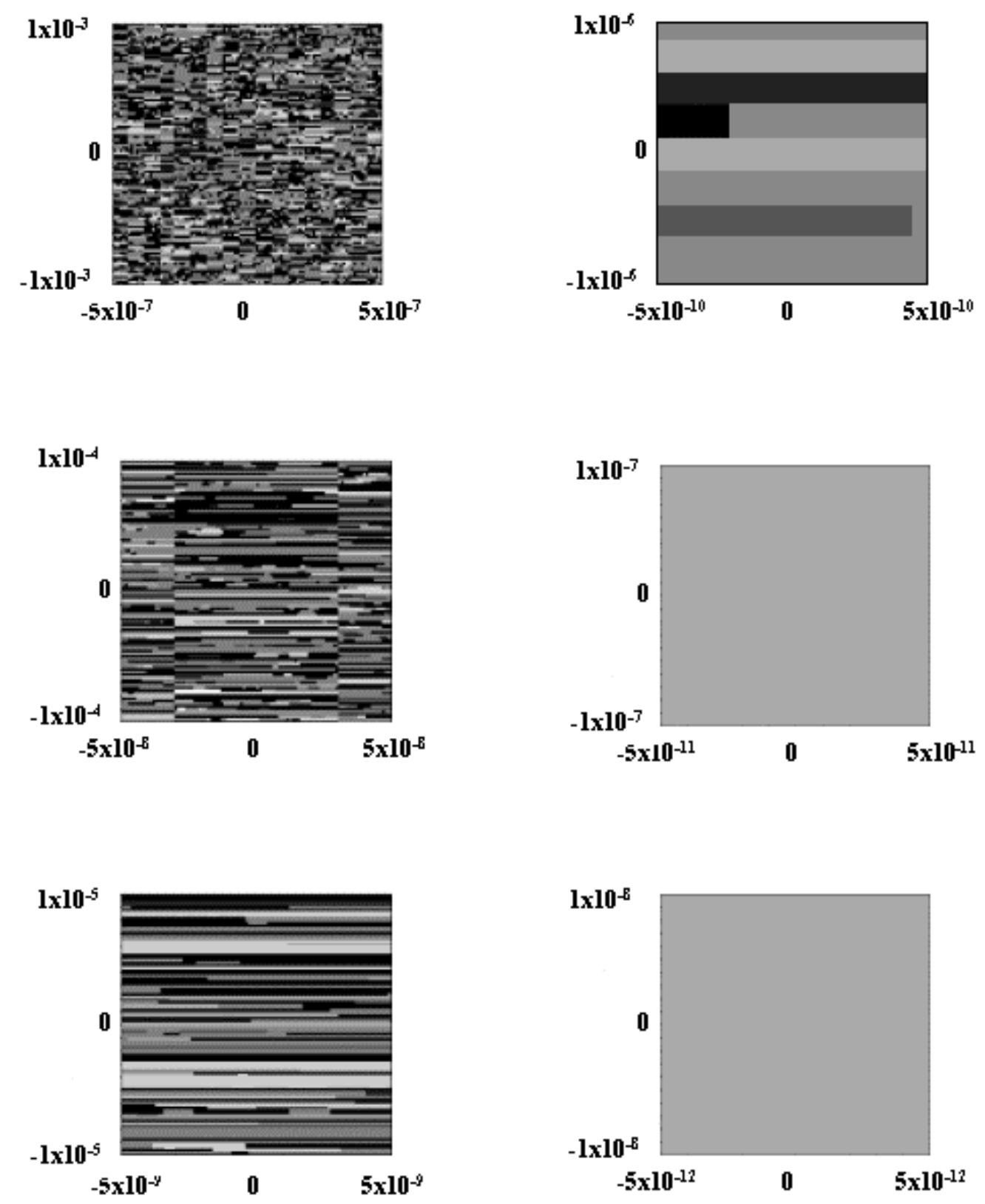

Figure 2. Kelly plots for ball 13 in the same format as in Figure 1, only with a further restricted range of initial conditions. Note the presence of vertical demarcations in the first two plots. The initial conditions ultimately restrict so that the plots show one uniform shade of gray, corresponding to the ball's landing in only one pocket. 

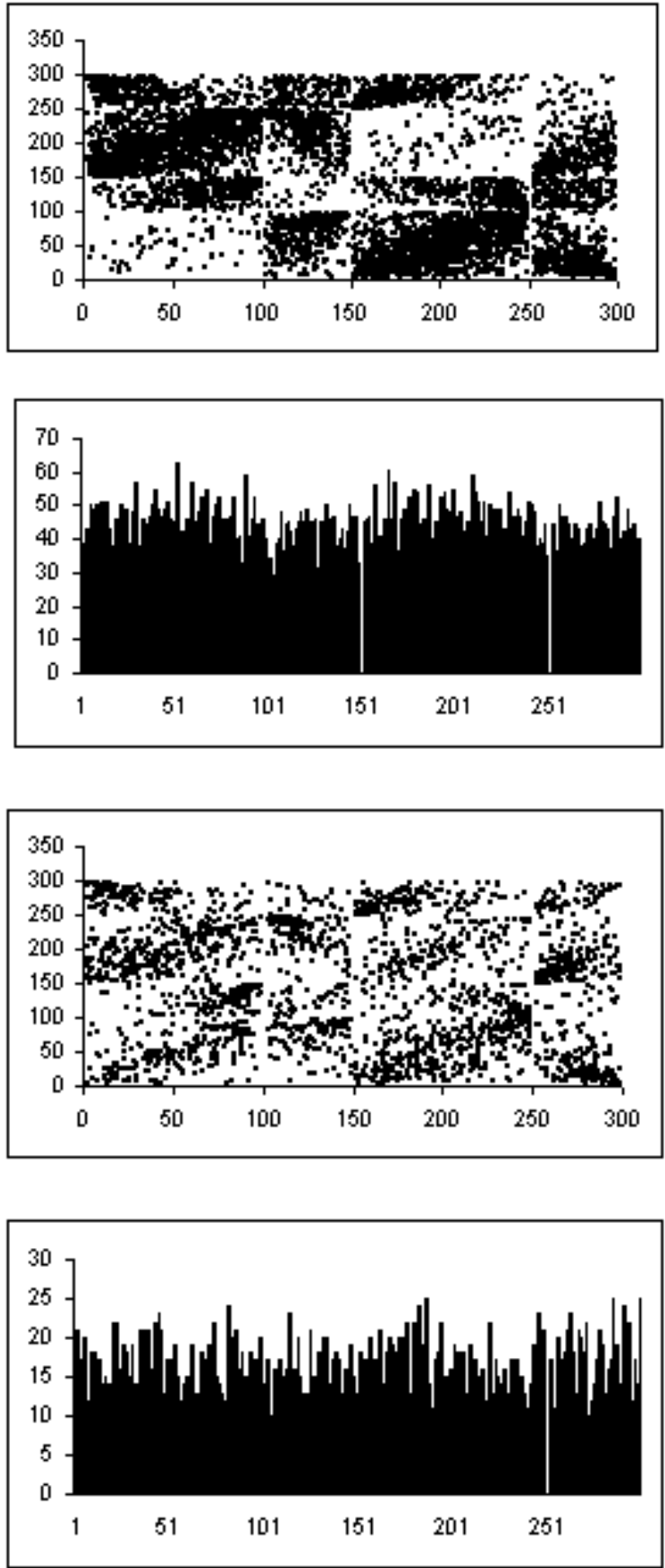

Figure 3. Return maps and histograms for $\mathrm{N}=3$ (top plots) and $\mathrm{N}=16$ (bottom plots). In the return maps, both axes are "pocket" number on the table perimeter; for the histograms, the vertical axis is reflection frequency and the horizontal axis is "pocket" number. 

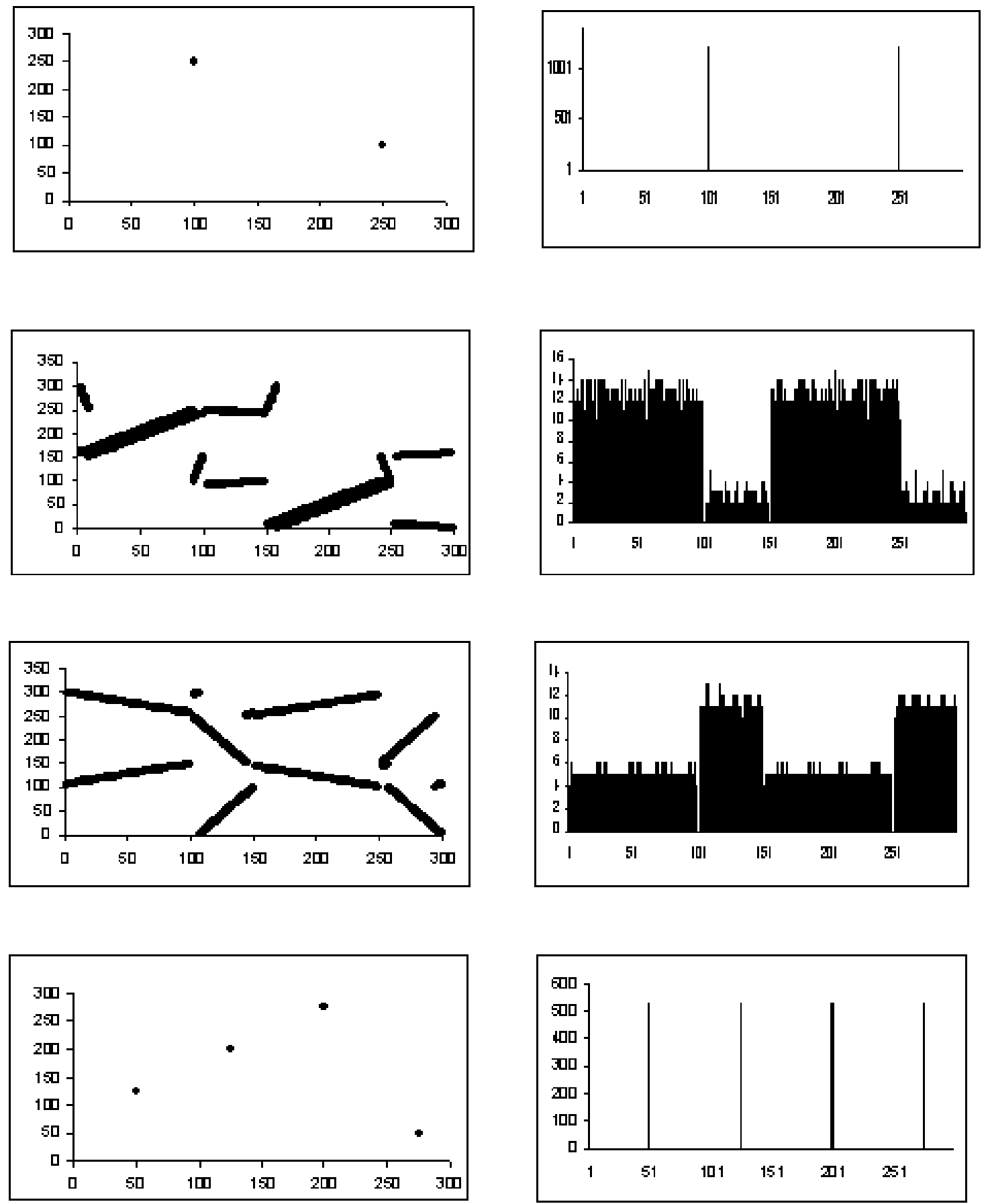

Figure 4. Return maps (left) and histograms (right) for simulations involving only the cue ball. The ball is directed straight downward, $0.001^{\circ}$ left of downward, $0.0001^{\circ}$ right of the middle of the bottom wall, and directly at the middle of the bottom wall (from top to bottom in the figure). Axis formats are the same as for those in Figure 3). 
corners and are consistent with the histograms. It is also apparent that, taking the plot densities into account, the diagonal for $\mathrm{N}=3$ is much less populated compared to that for $\mathrm{N}=16$. This is the case because the diagonal represents those collisions in which a ball strikes a pocket, reflects off another ball and hits the same pocket again. Clearly such an event is much less likely for $\mathrm{N}=3$ than for $\mathrm{N}=16$. From all the return maps we conclude that long-time memory (history) is important for the systems examined.

\section{c. Simulating only the cue ball}

Figures 4-6 provide detailed information about the dynamics of the cue ball in the absence of any others. Both the return map and histogram show that when hit straight down the ball is in a 2-cycle. In this case, the coordinate $(125,275)$ for the return map means the ball started at point 125 and moved in a straight line across the table to point 275 . It then moved from point 275 back to point 125 . The figure is of one ball moving back and forth alone on the table. Then, when the angle of its initial velocity is changed slightly it would appear that the system exhibits noisy four-cycles of various types, even when hit just short of the middle of the bottom table wall. When hit directly at the middle of the table bottom the ball is then locked in a 4-cycle. Then, sensitivity to initial conditions once again comes into play as the ball exhibits noisy four-cycles until it is hit directly to the lower left corner of the table, where it shows a three-cycle. One must be very careful to interpret the plots, however, bearing in mind that each corner is comprised of two table segments, or "pockets". So each corner registers two points and the side registers only one. Then, strong sensitivity to initial conditions is exhibited as the pattern of the ball becomes noisy until it is hit directly to the middle of the left wall of the table, undergoing a 2-cycle again. Based upon the appearance of the return maps we conclude that long-term memory is much less important for the cue ball alone than for cases where multiple balls are present.

The bifurcation plot in Figure 7 shows not only that the cue ball dynamics depend strongly on initial angle but that the greatest sensitivity occurs for angles closest to $\vartheta_{0}=0^{\circ}$. This makes sense because at $\vartheta_{0}$ $=0^{\circ}$ the system is locked in a two-cycle and immediately changes behavior to a noisy two-cycle, even for the smallest values of $\vartheta_{0}$ we use.

Although it is desirable to further analyze the return maps and certain Fourier transforms of other plots to understand the frequency behavior of the systems in question we certainly see that apparently the dynamics of even a one-ball system are extremely sensitive to initial conditions. In addition, our studies with only one ball prove very useful in validating the return maps and provide evidence that our algorithm for collisions does not produce significant numerical round off in the timescale of interest. We emphasize, however, that finite simulation time is a strong hindrance to proper (let alone absolute) interpretation of computer simulation results, and a finite time effects analysis would be most useful in determining, for example, if the system is really showing noisy 4-cycles or will ultimately show other types of behavior.

Conclusion

As mentioned in detail and enumerated previously, we conclude that the systems presented in this paper show chaotic behavior. The results and interpretations of our study agree with the work of Giordano, who conducts billiard simulations with the intent of detecting chaos. ${ }^{9}$ Several different table shapes are examined, including circles, squares and "stadium". The stadium shape is formed by two semicircles joined by straight segments of varying length. Phase space plots and divergence of the trajectories of two balls started out very close to one another are used to conclude that only for highly symmetric table shapes (square and circle for example) is the motion of a billiard nonchaotic. Even for tables that are stadiumshaped to the smallest degree, and certainly for those shapes that approximate traditional pool tables as used in our simulations, chaotic behavior is observed.

\section{ACKNOWLEDGEMENTS}

The authors are grateful to the referees for helpful comments. 

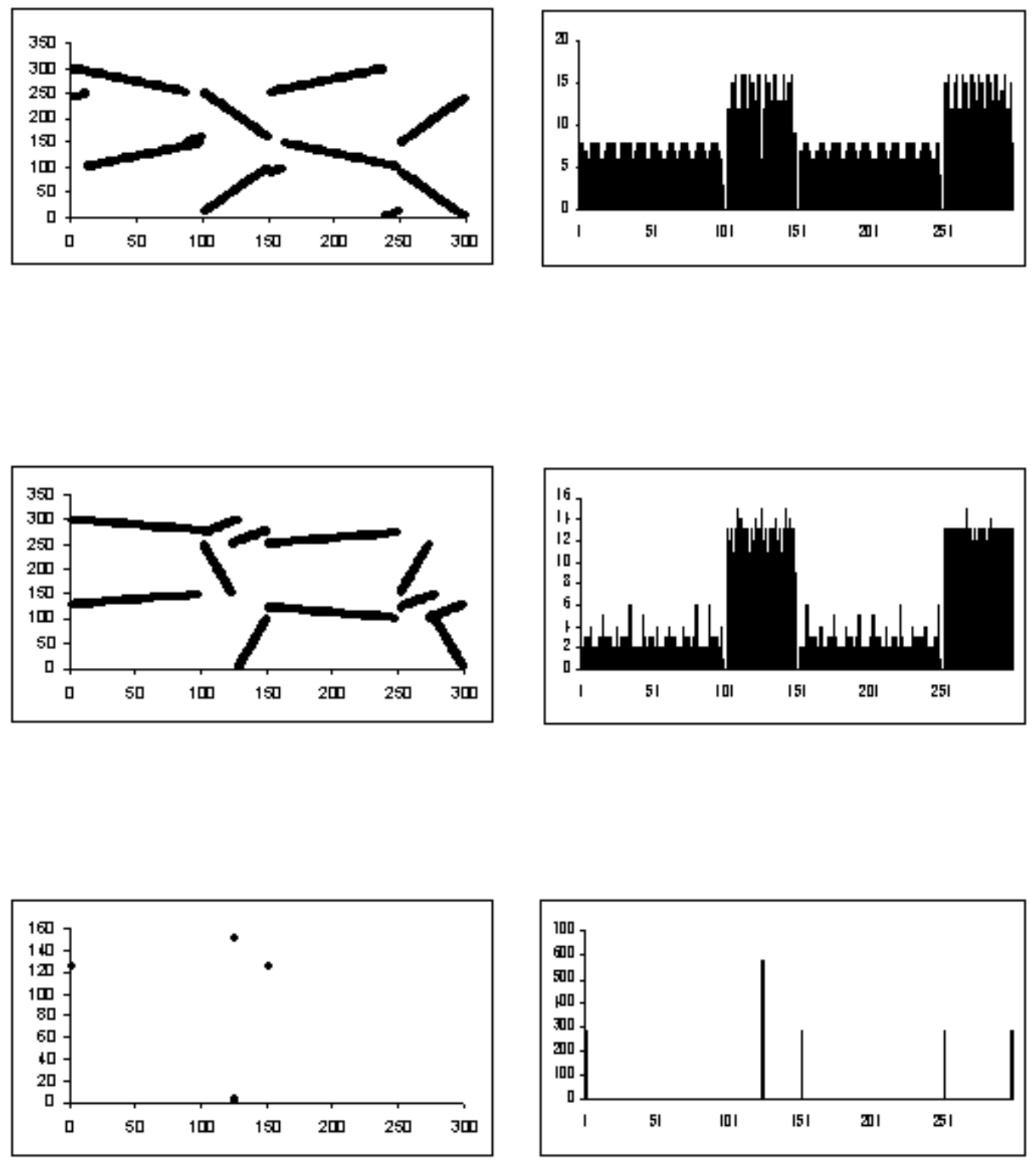

Figure 5. Return maps (left) and histograms (right) for simulations involving only the cue ball. The ball is directed $0.001^{\circ}$ to the left of the middle of the bottom wall, $0.0001^{\circ}$ to the right of the lower left corner, and directly at the lower left corner (from top to bottom in the figure). Axis formats are the same as for those in Figure 3. 

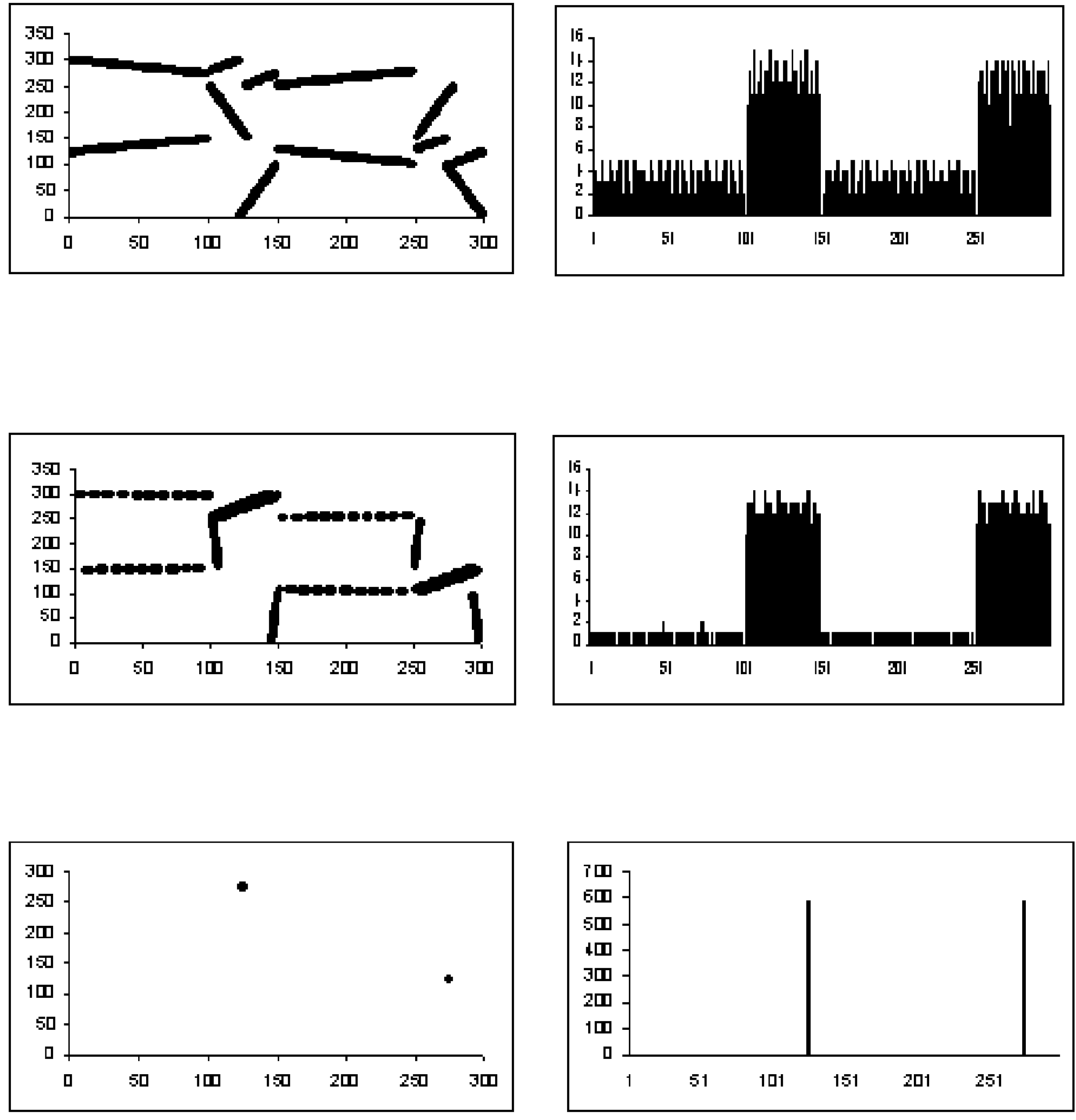

Figure 6. Return plots (left) and histograms (right) for simulations involving only the cue ball. The ball is directed $0.001^{\circ}$ above lower left corner, $0.0001^{\circ}$ below the middle of the left wall and directly at the middle of the left wall (from top to bottom in the figure). Axis formats are the same as for those in Figure 3. 


\section{REFERENCES}

1. Yale University, classes.yale.edu/ fractals/aut02/aut02daily.html

2. GTK Pool, www.gtkpool.seul.org

3. Virtual Pool, www.vpool.com

4. Ultimate Pool, www.macupdate.com/ info.php/id/5175

5. BilliardGL, www.tobias-nopper.de/ BillardGL / index-en.html
6. J. Moeller, Physics Dept., University of Northern lowa (unpublished).

7. Stadium Billiards, serendip.brynmawr. edu/chaos/doc.html

8. M.P Allen and D.J. Tildesley, Computer Simulation of Liquids, Oxford [England]: Clarendon Press ; New York: Oxford University Press, 1989, c1987.

9. Nicholas J. Giordano, Computational Physics, Prentice Hall, 1997 (pp. 67-73).

\section{University of Northern lowa}
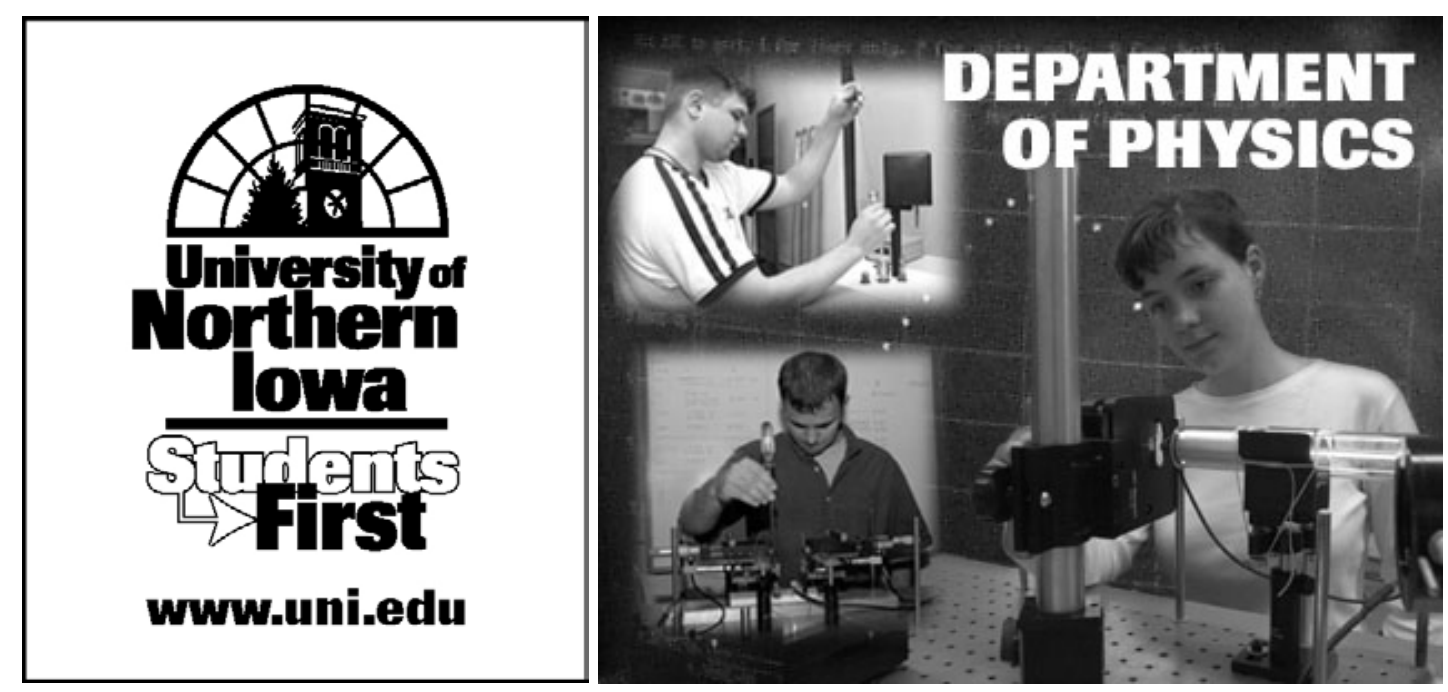


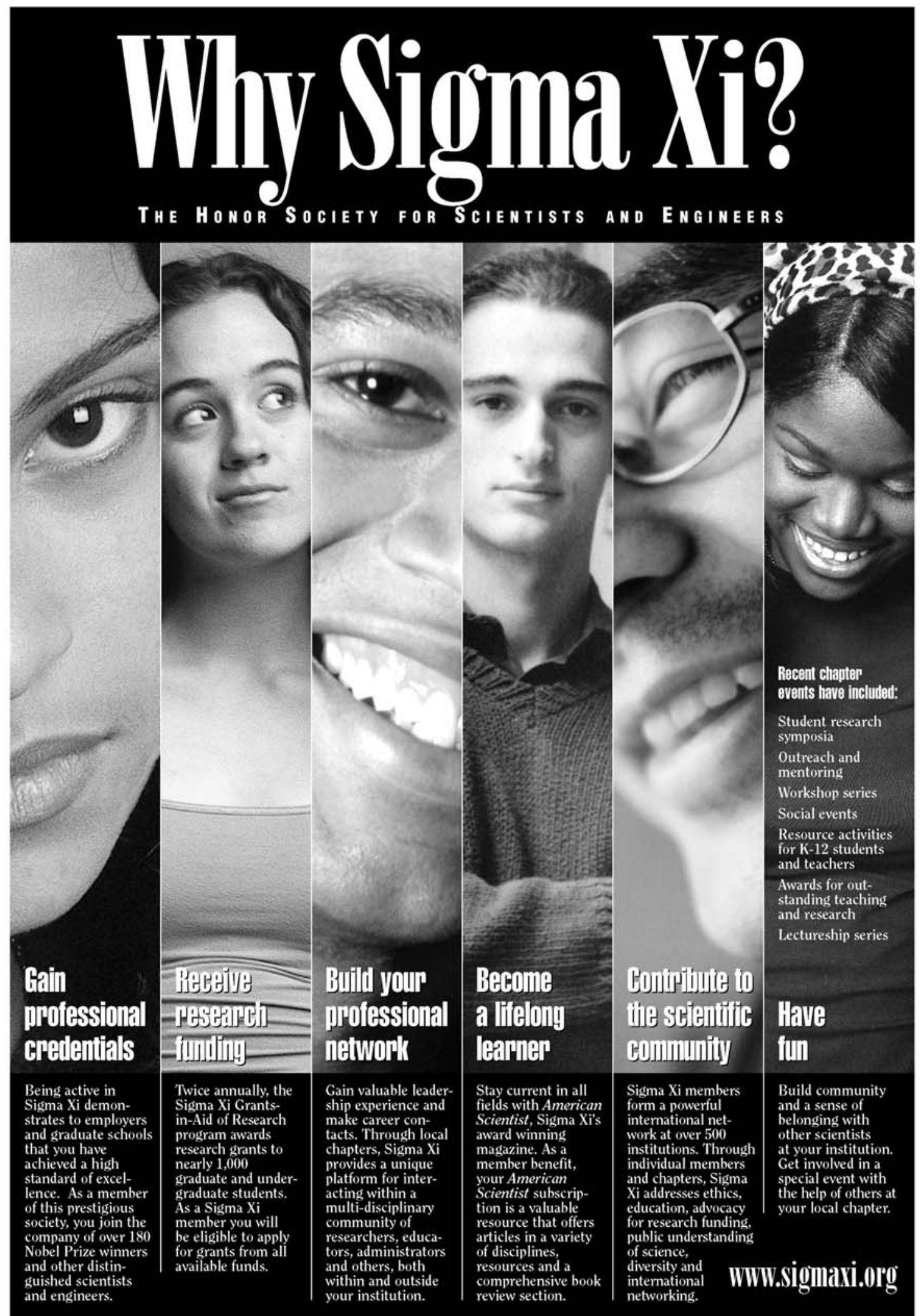

Sigma Xi, The Scientific Research Society • 88 Alexander Drive • P.O. Box 13975 • Research Triangle Park, NC 27708 • 818-549-4691 • 800-243-6534 